\title{
An ultraluminous supersoft source with a 4 hour modulation in NGC 4631
}

\author{
S. Carpano ${ }^{1}$, A. M. T. Pollock ${ }^{1}$, A. R. King ${ }^{2}$, J. Wilms ${ }^{3}$, and M. Ehle ${ }^{1}$ \\ 1 XMM-Newton Science Operations Centre, ESAC, ESA, PO Box 50727, 28080 Madrid, Spain \\ e-mail: scarpano@sciops.esa.int \\ 2 Department of Physics and Astronomy, University of Leicester, Leicester LE1 7 RH, UK \\ 3 Dr. Remeis-Observatory, Astronomisches Institut der FAU Erlangen-Nürnberg, Sternwartstr. 7, 96049 Bamberg, Germany
}

Received 4 April 2007 / Accepted 6 July 2007

ABSTRACT

\begin{abstract}
Context. Supersoft X-ray sources (SSSs) are characterised by very low temperatures $(<100 \mathrm{eV})$. Classical SSSs have bolometric luminosities in the range of $10^{36}-10^{38} \mathrm{erg} \mathrm{s}^{-1}$ and are modelled with steady nuclear burning of hydrogen on the surfaces of white dwarfs. However, several SSSs have been discovered with much higher luminosities. Their nature is still unclear.

Aims. We report the discovery of a $4 \mathrm{~h}$ modulation for an ultraluminous SSS in the nearby edge-on spiral galaxy NGC 4631, observed with XMM-Newton in 2002 June. Temporal and spectral analysis of the source is performed.

Methods. We use a Lomb-Scargle periodogram analysis for the period search and evaluate the confidence level using Monte-Carlo simulations. We measure the source temperature, flux and luminosity through spectral fitting.

Results. A modulation of $4.2 \pm 0.4 \mathrm{~h}$ ( $3 \sigma$ error) was found for the SSS with a confidence level $>99 \%$. Besides dips observed in the light curve, the flux decreased by a factor of 3 within $\sim 10 \mathrm{~h}$. The spectrum can be described with an absorbed blackbody model with $k T \sim 67 \mathrm{eV}$. The absorbed luminosity in the $0.2-2 \mathrm{keV}$ energy band was $2.7 \times 10^{38} \mathrm{erg} \mathrm{s}^{-1}$ while the bolometric luminosity was a hundred time higher $\left(3.2 \times 10^{40} \mathrm{erg} \mathrm{s}^{-1}\right)$, making the source one of the most luminous of its class, assuming the best fit model is correct.

Conclusions. This source is another very luminous SSS for which the standard white dwarf interpretation cannot be applied, unless a strong beaming factor is considered. A stellar-mass black hole accreting at a super Eddington rate is a more likely interpretation, where the excess of accreted matter is ejected through a strong optically-thick outflow. The $4 \mathrm{~h}$ modulation could either be an eclipse from the companion star or the consequence of a warped accretion disk.
\end{abstract}

Key words. galaxies: individual: NGC 4631 - X-rays: galaxies - X-rays: binaries

\section{Introduction}

The X-ray binaries known as supersoft sources (SSSs) are characterised by very soft emission with temperatures $<100 \mathrm{eV}$ and bolometric luminosities exceeding $10^{36} \mathrm{erg} \mathrm{s}^{-1}$. The standard model for such soft and high luminosities was proposed by van den Heuvel et al. (1992) as nuclear burning of hydrogen on the surface of a white dwarf of mass in the range $0.7-1.2 M_{\odot}$. On the other hand, if luminosities greatly exceed the Eddington limit for solar mass compact objects, it is not obvious that this type of model could apply unless beaming is considered. Several such very luminous SSSs have been reported in nearby galaxies: two in M 101 (Di Stefano \& Kong 2003; Kong et al. 2004; Mukai et al. 2005), one in M 51 (Di Stefano \& Kong 2003), one in M 81 (Swartz et al. 2002), one in the Antennae (Fabbiano et al. 2003) and two in NGC 300 (Kong \& Di Stefano 2003; Carpano et al. 2006).

In one of these SSSs, a short period of $5.4 \mathrm{~h}$ has been reported: the source located in the face-on spiral galaxy NGC 300, which was discussed by Kong \& Di Stefano (2003) and Carpano et al. (2006). The modulation was only present during a single XMM-Newton observation but was not visible 6 days earlier, when the source was a few times brighter. In this Letter, we report the discovery of an even more luminous SSS with a slightly shorter modulation, located in the nearby edge-on galaxy NGC 4631.

NGC 4631 is a SBc/d type galaxy located at an assumed distance of 7.5 Mpc (Golla \& Hummel 1994), with low galactic foreground absorption $\left(N_{\mathrm{H}}=1.2 \times 10^{20} \mathrm{~cm}^{-2}\right.$; Dickey \& Lockman 1990). A study of the giant diffuse X-ray emitting corona around the galaxy was performed by Wang et al. (2001) with Chandra and by Tüllmann et al. (2006) with XMM-Newton. The point-source population viewed by ROSAT was studied by Vogler \& Pietsch (1996) and Read et al. (1997). They reported the detection of 7 sources, including the supersoft source described in this Letter. Spectral fitting of this SSS using a bremsstrahlung model (Read et al. 1997) gave a temperature of $0.1 \mathrm{keV}$ with high $\left(8 \times 10^{21} \mathrm{~cm}^{-2}\right)$ intrinsic absorption; the corresponding absorbed luminosity in the $0.1-2.0 \mathrm{keV}$ energy band was of $1.9 \times 10^{38} \mathrm{erg} \mathrm{s}^{-1}$. In the long-term light curve (Vogler \& Pietsch 1996) the source was not visible in two of six data sets although, because the source flux was close to the detection limit in the other four, time variability could not be established.

In this Letter, we report the discovery of a $4 \mathrm{~h}$ modulation for the ultraluminous SSS in NGC 4631. The remainder of the Letter is organised as follows. Section 2 describes the XMM-Newton observation and data reduction. In Sect. 3, we present timing and spectral analysis of the SSS, reserving discussion for Sect. 4. 


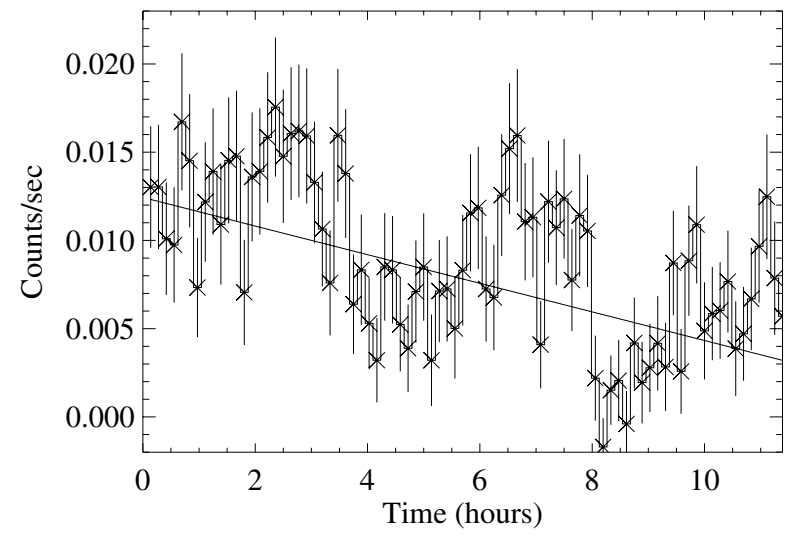

Fig. 1. Mean XMM-Newton EPIC-MOS and pn background-subtracted light curve of the SSS in NGC 4631. Error bars are at $1 \sigma$. The straight line shows the best linear fit to the lightcurve.

\section{Observation and data reduction}

NGC 4631 was observed by XMM-Newton on 2002 June 8 for $55 \mathrm{ks}$. The EPIC-MOS (Turner et al. 2001) and EPIC-pn (Strüder et al. 2001) cameras were operated in full frame mode with the medium MOS filter and the thin pn filter. After screening the MOS data for proton flares using standard procedures ${ }^{1}$, a total of 45 and $37 \mathrm{ks}$ of low-background emission remained for the MOS and pn, respectively.

Using the XMM-Newton Software Analysis System (SAS) edetect_chain task, which performs maximum-likelihood source detection, the SSS was detected with a maximum likelihood of 1919. The best-fit coordinates were: $\alpha_{\mathrm{J} 2000}=$ $12^{\mathrm{h}} 42^{\mathrm{m}} 16^{\mathrm{s}} .1$ and $\delta_{\mathrm{J} 2000}=+32^{\circ} 32^{\prime} 49^{\prime} .5$ with a statistical error of 0.3 .

\section{Timing and spectral analysis of the SSS}

The combined EPIC-MOS and pn background-subtracted lightcurve is shown in Fig. 1, with a time bin size of $500 \mathrm{~s}$. Times are given in hours from the start of the observation. Periods of high background at the end of the observation have been excluded from the data. Two dips are apparent in the lightcurve in addition to a long-term decrease by a factor of 3 (Fig. 1, straight line).

We performed a period search with Lomb-Scargle periodogram analysis (Lomb 1976; Scargle 1982) after having removed the general trend of the decreasing flux. Confidence levels were measured by means of Monte Carlo simulations assuming a null hypothesis of white noise. Results are shown in Fig. 2. We found that the $4.17 \mathrm{~h}$ period is significant at $>99 \%$ confidence. This modulation, although less significant, is also present in the MOS data alone. We estimated the uncertainty of the period by fitting a sine function using the IDL $^{2}$ task curvefit to the detrended light curve. The best-fit period by this method was $4.11_{-0.34}^{+0.40} \mathrm{~h}$, close to the value from the Lomb-Scargle analysis. The errors quoted are at the $3 \sigma$ level.

The corresponding light curve folded at $4.17 \mathrm{~h}$ is shown in Fig. 3. The shape is slightly asymmetric, the flux change before the dip sharper than afterwards. The dip is also relatively narrow.

\footnotetext{
${ }^{1}$ http://xmm.esac.esa.int/external/xmm_user_support/ documentation/sas_usg/USG/

${ }^{2}$ http://www.ittvis.com/idl
}

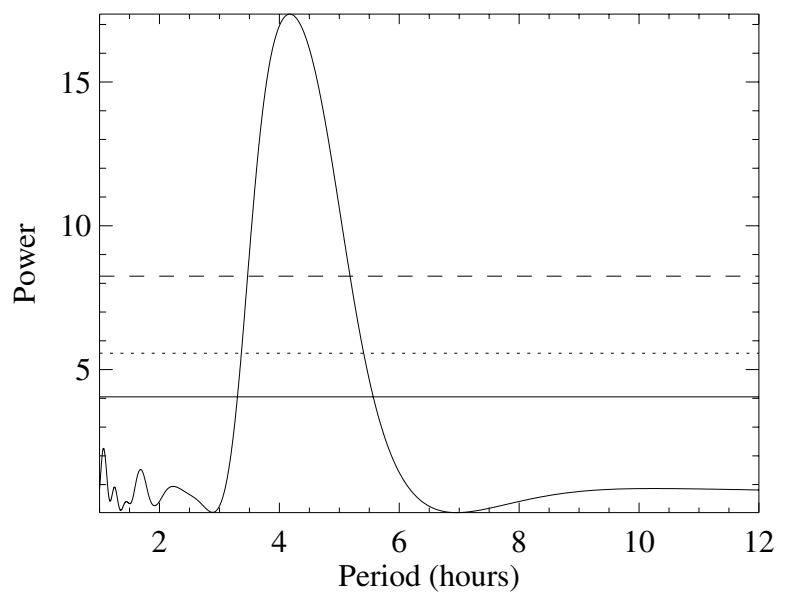

Fig. 2. Search for periodicities in the XMM-Newton light curve of the NGC 4631 SSS using Lomb-Scargle periodogram analysis, after removing the decreasing flux trend. The full, dotted and dashed lines represent the $68 \%, 90 \%$ and $99 \%$ confidence levels, respectively, as determined from Monte Carlo simulations.

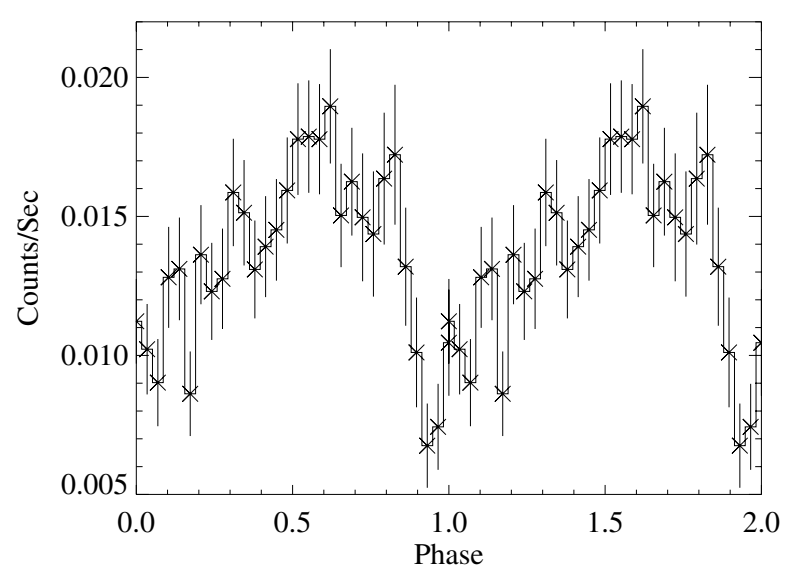

Fig. 3. XMM-Newton light curve of the NGC $4631 \mathrm{SSS}$ folded at $4.17 \mathrm{~h}$ using 30 bins, after removing the decreasing flux trend.

Figure 4 shows the pn and MOS spectra of the SSS. The data are binned to have at least 25 counts in each energy bin. Almost no flux is visible above $2 \mathrm{keV}$. We first tried to model the data with an absorbed single blackbody, yielding $\chi_{v}^{2}=1.95$. From the residuals shown in Fig. 4, some excess around $0.85 \mathrm{keV}$ is observed. Adding a Gaussian line significantly improved the fit, resulting in a reduced $\chi_{v}^{2}=1.22$ for $v=53$ degrees of freedom. The absorbed bremsstrahlung model used by Read et al. (1997), with or without a Gaussian line, does not describe the data well $\left(\chi_{v}^{2}=3\right)$. We also tried to fit a black body with a power-law to describe the continuum but the normalisation constant of this last component is very small and spectral parameters as well as luminosities do not change significantly (less than a factor of 2). Alternatively to the Gaussian emission line at $0.8 \mathrm{keV}$, an absorption edge could also describe the spectrum $\left(\chi_{v}^{2}=1.35\right)$. Unfortunately due to the low statistic of the data above $1 \mathrm{keV}$, the absorption depth parameter cannot be constrained. Absorption edges have been reported by Swartz et al. (2002) and Kong et al. (2004) for the luminous SSSs in M 81 and M 101, respectively, indicating the presence of a warm absorber in the vicinity of the X-ray source. The best-fit parameters of the absorbed combined blackbody and single Gaussian-line model are shown in Table 1. $N_{\mathrm{H}}$ is the equivalent column density of neutral hydrogen, $k T$ the temperature, $E_{\mathrm{L}}$ the energy of the line and $\sigma_{\mathrm{L}}$ its width. The 


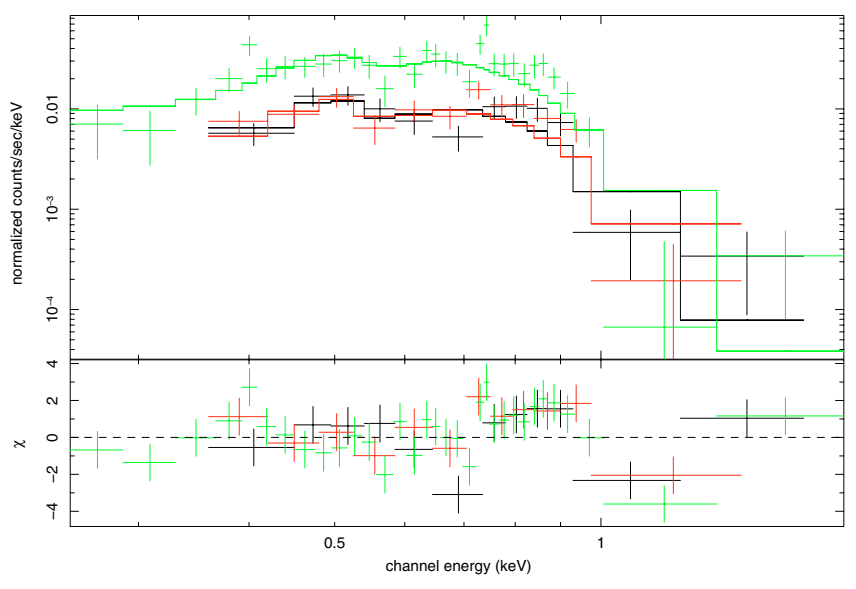

Fig. 4. XMM-Newton EPIC-pn and MOS spectra of the SSS in NGC 4361 and the best-fit spectral model with the apparent line emission. Bottom: residuals expressed in $\sigma$.

Table 1. Results of the spectral fits for the SSS in NGC 4361, using an absorbed blackbody model and a Gaussian line (phabs*(bbody+gauss), in XSPEC).

\begin{tabular}{ll}
\hline \hline Parameter & Value (units) \\
\hline$N_{\mathrm{H}}$ & $4.94_{-1.64}^{+1.61} \times 10^{21}\left(\mathrm{~cm}^{-2}\right)$ \\
$k T$ & $67_{-15}^{+5}(\mathrm{eV})$ \\
$\mathrm{E}_{\mathrm{L}}$ & $0.80_{-0.05}^{+0.03}(\mathrm{keV})$ \\
$\sigma_{\mathrm{L}}$ & $0.07_{-0.02}^{+0.04}(\mathrm{keV})$ \\
$F_{0.2-2 \mathrm{keV}}$ & $3.97_{-1.57}^{+4.76} \times 10^{-14}\left(\mathrm{erg} \mathrm{s}^{-1} \mathrm{~cm}^{-2}\right)$ \\
$L_{0.2-2 \mathrm{keV}}^{\text {obs }}$ & $2.67_{-1.06}^{+3.20} \times 10^{38}\left(\mathrm{erg} \mathrm{s}^{-1}\right)$ \\
$L_{0.2-2 \mathrm{keV}}^{\text {unabs }}$ & $1.99_{-1.27}^{+0.59} \times 10^{40}\left(\mathrm{erg} \mathrm{s}^{-1}\right)$ \\
$L_{\text {bol }}$ & $3.22_{-2.88}^{+1.35} \times 10^{40}\left(\mathrm{erg} \mathrm{s}^{-1}\right)$ \\
\hline
\end{tabular}

corresponding 0.2-2 kev flux, absorbed, unabsorbed and bolometric luminosities are shown in the last four rows. Uncertainties are given at the $90 \%$ confidence level, except for the bolometric luminosity at $99 \%$. As the source was close to a pn CCD gap, the flux and luminosities were calculated from the MOS data alone.

Our data modelling shows the source to have a very high intrinsic absorption column giving a bolometric luminosity that makes the SSS one of the most luminous of its class. Such high absorption could be due to a strong intrinsic absorption in the vicinity of the emitting source or due to gas of the hosting galaxy since it is edge-on oriented. The source is also highly variable. The object has also been observed once with Chandra, about two years before the XMM-Newton observation, on 2000 April 16 for $59 \mathrm{ks}$ when 31 net counts were detected. Converting this rate to flux using WebPIMMS ${ }^{3}$ with the spectral parameters of Table 1, we estimate that the mean observed luminosity at that time in the $0.2-2 \mathrm{keV}$ energy band was $1.7 \times 10^{37} \mathrm{erg} \mathrm{s}^{-1}$. This is about a factor of 10 lower than the XMM-Newton measurement two years later and the earlier ROSAT value (Read et al. 1997).

\section{Discussion}

The SSS reported in this Letter is one of the most luminous of its class. Besides the bright and soft X-ray emission, the source is particular for its $4 \mathrm{~h}$ modulation superimposed on a decreasing flux trend during the XMM-Newton observation.

\footnotetext{
${ }^{3}$ http://heasarc.gsfc.nasa.gov/Tools/w3pimms.html
}

On only one other occasion, for a SSS in NGC 300 (Kong \& Di Stefano 2003), has a short modulation been reported for such a bright SSS, of $5.4 \mathrm{~h}$ in that case, in the one of the two XMMNewton observations in which the source was fainter. A discussion of the source has also been reported by Carpano et al. (2006).

The high luminosity $\left(>10^{40} \mathrm{erg} \mathrm{s}^{-1}\right)$ of the NGC $4361 \mathrm{SSS}$, which exceeds the Eddington luminosity for a white dwarf or a neutron star by a factor of 100 , might be due to a strong wind or outflow of a stellar-mass black hole accreting at a super Eddington rate. This idea has also been suggested by Fabbiano et al. (2003) and Mukai et al. (2005) for the ultraluminous SSSs in the Antennae and M 101, respectively. The model has been reconsidered in more detail by King \& Pounds (2003), as summarised below. Other less convincing models in the literature, as summarised by Carpano et al. (2006), have variously involved strong beaming from steady nuclear burning of hydrogen at the surface of a white dwarf; accretion onto an intermediate-mass black hole; or soft emission of an accretion disk observed at high inclination.

King \& Pounds (2003) suggested that a black hole accreting at super-Eddington rate, ejects the excess matter through a strong wind or outflow that is likely to be optically thick to electron scattering for $\dot{M}_{\text {out }} \sim \dot{M}_{\text {Edd }}$ with a photospheric radius of the order of few tens of the Schwarzschild radius of the black hole. The outflow is radial and occupies a double cone of solid angle $4 \pi b$, where the beaming factor $b$ can be either $\sim 1$ or $\ll 1$. The emission line present in the spectrum of the SSS in NGC 4631, which is likely to be composed of several unresolved lines, or alternatively the absorption edge, might come from optically thin parts of the matter outflow. These could either be near the edges of the conical outflow, or the leading edge of the outflow if this is time-dependent. The temperature resulting from the black body emission is (King \& Pounds 2003):

$T_{\text {eff }}=1 \times 10^{5} g^{-1 / 4} \dot{M}_{1}^{-1} M_{8}^{3 / 4} \mathrm{~K}$

where $g(b)=1 / b$ or $1 /\left(2 b^{1 / 2}\right)($ for $b \sim 1$ or $b \ll 1), \dot{M}_{1}=$ $\dot{M}_{\text {out }} /\left(1 M_{\odot} \mathrm{yr}^{-1}\right)$, and $M_{8}=M / 10^{8} M_{\odot}$, and $M$ is the mass of the accretor.

Assuming the source luminosity is close to the Eddington limit, $\dot{M}_{\text {out }} \sim \dot{M}=L /\left(\eta \mathrm{c}^{2}\right)$, where $L$ is the luminosity and $\eta$ the radiative efficiency, we are able to estimate the mass of the black hole

$M=0.27\left[\frac{g^{1 / 4}}{\eta}\right]^{4 / 3} \quad M_{\odot}$.

As $g^{1 / 4}$ is of order unity unless $b \lesssim 0.1$, for a typical value of $\eta \sim 0.1$, the bolometric luminosity of $3.2 \times 10^{40} \mathrm{erg} \mathrm{s}^{-1}$ gives a corresponding black-hole mass of $6 M_{\odot}$ and an accretion rate of $5.6 \times 10^{-7} M_{\odot} \mathrm{yr}^{-1}$. Such an accretion rate can easily be achieved either by thermal-time-scale mass transfer after a highmass companion has filled its Roche Lobe or during outbursts of soft X-ray transients (King 2002).

The $4 \mathrm{~h}$ modulation could be caused either by an eclipse from the companion star or by the presence of a warped disk. This latter hypothesis is driven by the fact that the $5.4 \mathrm{~h}$ modulation in the SSS of NGC 300 was observed in only one of two XMM-Newton observations. Barnard et al. (2006) have recently reported that the X-ray binary Bo 158 in M 31, shows regular dips at $2.78 \mathrm{~h}$ but not in all of their observations. These authors suggested this dipping to be caused by an elongated precessing disk. 
To summarise, the ultraluminous SSS in NGC 4631 reported in this Letter may be an X-ray binary system with a short ( $\$ 10 \mathrm{~h})$ orbital period, where the high mass transfer rate is either due to a massive companion filling its Roche Lobe or because the system is in outburst. When matter is accreted above the Eddington rate, the excess matter is ejected via a strong, optically-thick outflow in which case a mass of $6 M_{\odot}$ can be estimated for the black-hole companion.

Acknowledgements. This paper is based on observations obtained with $X M M-N e w t o n$, an ESA science mission with instruments and contributions directly funded by ESA Member States and NASA.

\section{References}

Barnard, R., Foulkes, S. B., Haswell, C. A., et al. 2006, MNRAS, 366, 287 Carpano, S., Wilms, J., Schirmer, M., \& Kendziorra, E. 2006, A\&A, 458, 747

Dickey, J. M., \& Lockman, F. J. 1990, ARA\&A, 28, 215

Di Stefano, R., \& Kong, A. K. H. 2003, ApJ, 592, 884
Fabbiano, G., King, A. R., Zezas, A., et al. 2003, ApJ, 591, 843

Golla, G., \& Hummel, E. 1994, A\&A, 284, 777

King, A. R. 2002, MNRAS, 355, L13

King, A. R., \& Pounds, K. A. 2003, MNRAS, 345, 657

Kong, A. K. H., \& Di Stefano, R. 2003, ApJ, 590, L13

Kong, A. K. H., Di Stefano, R., \& Yuan, F. 2004, ApJ, 617, L49

Lomb, N. R. 1976, Ap\&SS, 39, 447

Mukai, K., Still, M., Corbet, R. H. D., Kuntz, K. D., \& Barnard, R. 2005, ApJ, 634, 1085

Read, A. M., Ponman, T. J. \& Strickland, D. K. 1997, MNRAS, 286, 626

Scargle, J. D., 1982, 263, 835

Strüder, L., Briel, U., Dennerl, K., et al. 2001, A\&A, 365, L18

Swartz, D. A., Ghosh, K. K., Suleimanov, V., Tennant, A. F., \& Wu, K. 2002, ApJ, 574, 382

Tüllmann, R., Breitschwerdt, D., Rossa, J., Pietsch, W., \& Dettmar, R.-J. 2006, A\&A, 457, 779

Turner, M. J. L., Abbey, A., Arnaud, M., et al. 2001, A\&A, 365, L27

van den Heuvel, E. P. J., Bhattacharya, D., Nomoto, K., \& Rappaport, S. A. 1992, A\&A, 262, 97

Vogler, A., \& Pietsch, W. 1996, A\&A, 311, 35

Wang, Q. D., Immler, S., Walterbos, R., Lauroesch, J. T., \& Breitschwerdt, D. 2001, ApJ, 555, L99 